\title{
Management of patients with an incomplete cervical spinal cord injury
}

\author{
T Asazuma, K Satomi, N Suzuki, Y Fujimura and K Hirabayashi \\ Department of Orthopaedic Surgery, Keio University, School of Medicine, 35 Shinanomachi, Shinjuku-ku, Tokyo 160, \\ Japan
}

\begin{abstract}
Forty-five patients with incomplete cervical spinal cord injuries were examined as to the mechanism of injury, neurological deficits in the initial stage and differences in treatment. Nineteen $(42.2 \%)$ were treated non-surgically and $26(57.8 \%)$ surgically. Their injuries could be divided into seven types of cervical spinal cord injury according to Usui's classification. Neurological changes were evaluated with Frankel's grading system. The neurological prognosis was relatively better in those with a unilateral cord injury type, but there was no statistical difference. Thirty-seven patients $(82.2 \%)$ showed neurological improvement of at least one Frankel grade. There was no statistical difference between the surgical and nonsurgical groups regarding neurological improvement, but all who underwent early surgery (within 4 weeks of being injured) improved. Surgical treatment was considered to be indicated for patients with cervical spinal canal narrowing, when satisfactory neurological improvement is not obtained by conservative treatment.
\end{abstract}

Keywords: cervical spine; incomplete spinal cord injury; cervical spinal stenosis; surgical treatment; neurological prognosis

\section{Introduction}

Spinal paralysis is known to improve to a varying degree in patients with an incomplete cervical spinal cord injury (SCI). Very few studies refer to correlations between the extent of improvement, the mechansim of injury, the initial neurological state, and differences in treatment. There has not been a clear consensus in previous reports on the management of this type of lesion. Our purpose was to clarify the neurological prognosis, and to establish a type of management for those with an incomplete cervical SCI.

\section{Materials and methods}

Between 1965 and 1991, 45 patients (38 men and seven women) who had an incomplete traumatic cervical SCI were treated surgically at Keio University Hospital. The patient's ages ranged from 15 to 82 years (mean 51 years). The follow-up periods ranged from 1 year to 6 years and 11 months (mean, 1 year and 7 months). Twenty-five of the patients $(55.5 \%)$ were in their 5 th and 6th decades, whereas only one $(2.2 \%)$ was a teenager at the time of injury. Fifteen patients $(33.3 \%)$ experienced major trauma and $30(66.6 \%)$ minor trauma. A history of excess use of alcohol before the accident was found for eight of the 45 patients $(17.8 \%)$ whose SCI were mainly due to minor falls. Plain X-ray films, physical examinations, and knowledge regarding the type of accident were analyzed to determine the mechanism of injury. The location of head wounds

\footnotetext{
Correspondence: T Asazuma
}

may provide information on the mechanism of injury, and head or forehead wounds were present in 24 patients $(53.3 \%)$, forehead wounds being found in 15 , parietal wounds in seven and occipital wounds in two. Of the 45 patients studied, $22(48.9 \%)$ sustained extension injuries, four $(8.9 \%)$ axial compression and extension, and one $(2.2 \%)$ pure axial compression. The actual mechanism of injury could not be determined in 11.

There was neither bony injury nor dislocations in 25 of the patients $(55.6 \%)$, but, $12(26.7 \%)$ had an anterior displacement of less than half the vertebral body diameter in the plain lateral X-ray films. Five patients $(11.1 \%)$ had compression fractures and four $(8.9 \%)$ tear drop fractures. A spinous process fracture was seen in one patient, and a facet fracture in another $(2.2 \%)$ (three patients had both dislocations and fractures).

Plain X-rays also showed other abnormalities of the cervical spine which could be relevant. Twenty-four $(53.3 \%)$ had posterior osteophytes, $10(22.2 \%)$ had cervical canal stenosis (defined as below $12 \mathrm{~mm}$ in A-P diameter of the spinal canal), five $(11.1 \%)$ ossification of the posterior longitudinal ligament (OPLL) and one $(2.2 \%)$ congenital abnormalities (C2-3 synostosis). Posterior osteophytes were present at the level of C56 in 15 patients; at C5-6-7 in three, at C4-5-6 and $\mathrm{C} 5$ in each of two patients and at C6-7 and C6 in one patient. In terms of the neurological lesion level of the spinal cord, the C6 cord level was affected in 23 patients $(51.1 \%)$.

According to Usui and Hirabayashi, ${ }^{1}$ traumatic 


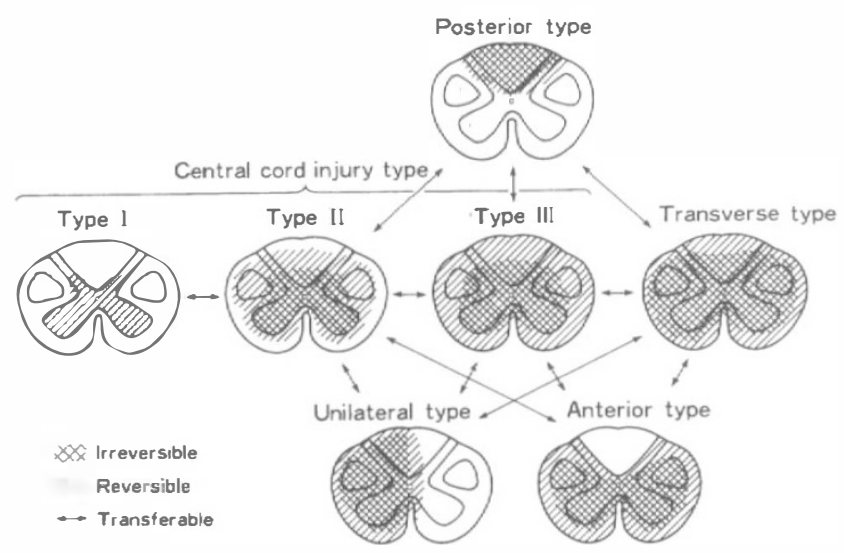

Figure 1 Traumatic incomplete cervical spinal cord injury as classified by Usui and Hirabayashi (1981)

incomplete cervical spinal cord injury is devisible into seven types: anterior cord injury, posterior cord injury, central cord injury and unilateral cord injury (Figure 1), central cord injury type having the three subtypes Types I, II and III. Central cord injury type has the following characteristics: the degree of paralysis is more severe in the upper than in the lower limbs immediately after injury and during the recovery period. Type I is characterized by no or only slight disturbance of the lower limbs from the onset of injury. In Type II, the degreee of paralysis is severe in all four limbs during the initial stage of injury. Disturbance in the lower limbs is expected to improve to some extent and improvement of the upper limbs is expected to occur later than in the lower limbs. In Type III, there is better improvement in the lower than in the upper limbs, but gait disturbance remains. Incomplete transverse cord injury type is included in this series.

The number of patients and degrees of cervical cord injury are shown as follows. Central cord injury was seen in 32 patients $(71.1 \%)$, Type I by 7 , Type II by 11 , and Type III by 14 . The unilateral cord injury type (Brown-Séquard type) in seven patients, and the transverse cord injury type in five. Only one patient had an anterior cord injury, and no patient had a posterior cord injury.

Associated injuries occurred in six of the 45 patients: Long bone fractures were seen in two patients, and a head injury with transient consciousness loss, Th12 spinal compression fracture, a chest injury, and an electric burn at left hand were seen in four patients respectively. No patient had multiple associated injuries and multiple spinal cord injury at different levels. These injuries did not significantly affect the management of the cervical lesion.

Twenty-nine patients received dexamethasone sodium phosphate $(8 \sim 16 \mathrm{mg} /$ day $)$ for 2 to 3 days on their admission. Nineteen patients $(42.2 \%)$ were treated non-surgically. Skull traction in 12, and
Glisson traction in six. Only one patient was treated with bed rest. Twenty-six patients $(57.8 \%)$ were treated surgically. Anterior decompression and fusion was performed in 16 patients, posterior decompression (expansive open door laminoplasty ${ }^{2}$ ) in nine and anterior decompression and fusion combined with expansive open door laminoplasty in one. Surgery took place from 2 days to 2 years and 3 months after injury (average 6.5 months). Eight patients underwent surgery within 4 weeks of injury (early stage surgery), and 18 patients more than 6 weeks after injury (late stage surgery).

Neurological change was evaluated by use of the Frankel grid $^{3}$ as it is a method widely used for representing the recovery of spinal cord injury patients. The data were analyzed statistically using Fisher's exact probability test.

\section{Results}

By definition, all of the patients had an incomplete cervical lesion. In all, 13 patients improved from grade $\mathrm{B}$ (having no motor power) to grade $\mathrm{D}$ (being able to walk), and 14 improved from grade $\mathrm{C}$ (some motor power but no functional ability to walk) to grade D. Five patients improved from grade $\mathrm{B}$ to $\mathrm{C}$, and five from grade $\mathrm{D}$ to $\mathrm{E}$ (full motor power and normal sensation). Frankel grades remained unchanged in eight patients at follow-up. No patient had neurological deterioration.

The Frankel grades at the initial and final assessments were analyzed for each injury type. Six patient $(85.7 \%)$ improved more than one grade in Type I, eight (72.7\%) improved in Type II, 11 (78.6\%) improved in Type III central cord injury. Four patients $(80 \%)$ improved more than one grade in transverse type and seven $(100 \%)$ improved in unilateral type. Four patients remained grade $\mathrm{C}$ in Type III and two still remained grade $\mathrm{C}$ in transverse type although all the patients were at the level of grade $\mathrm{D}$ or $\mathrm{E}$ in Type I, II central cord injuries and unilateral type at follow-up period. Neurological improvement was relatively better in the unilateral type although there was no statistical difference between each injury type (Figure 2).

Of the eight older patients (over 65 years), six had central cord type injuries and two transverse type. Five of these patients $(62.5 \%)$ improved more than one grade, but three with a Type III central cord injury or transverse injury were not able to walk (Frankel C) during the follow-up period. On the other hand, of the 37 young patients (under 64 years old), 35 (94.6\%) improved more than one grade. There was a statistical difference between the two groups $(P<0.01)$. The relationship between neurological recovery and the magnitude of the injury was examined. In the major trauma group, eight patients improved one grade, five improved two grades, and two did not improve; 13 patients $(86.7 \%)$ in all improved. In the minor trauma group, 18 patients improved one grade, seven 


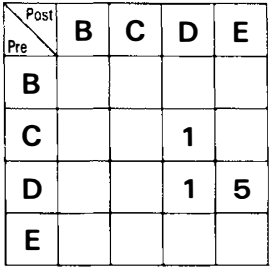

Type I $(n=7)$

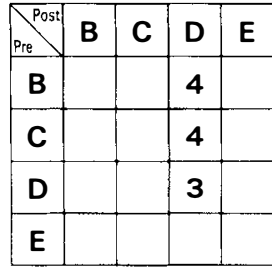

Type II $(n=11)$

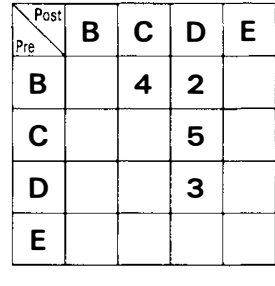

Type III $(n=14)$

Central spinal cord injuries

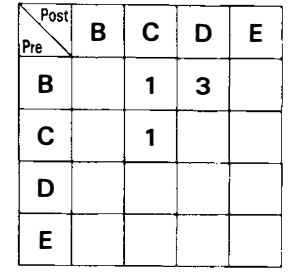

Transverse type $(n=5)$

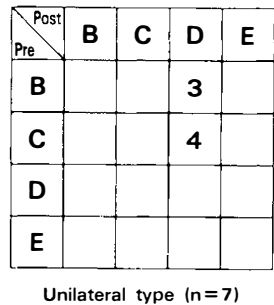

Unilateral type $(n=7)$
Figure 2 Neurological assessment based on the type of the injury

improved two grades and five did not improve; 25 patients $(83.3 \%)$ in all improved. There was no statistical difference between the major and minor trauma groups in terms of neurological recovery.

A comparison of the relationship between neurological recovery and the mechanism of injury showed no statistical difference between flexion and extension injuries.

The relationship between neurological recovery and canal narrowing factors was examined. Eight patients $(88.9 \%)$ improved who had a disc herniation, three $(60 \%)$ improved with OPLL and eight (80\%) improved in canal stenosis group. Patients with a disc herniation improved better than did those with OPLL $(P<0.05)$ (Figure 3$)$.

Clinical results were compared for the surgical and non-surgical treatments. Twenty-two patients $(84.6 \%)$ in the surgical treatment group, improved more than one grade, as did $15(78.9 \%)$ in the conservative treatment group. There was no statistical difference between the two groups.

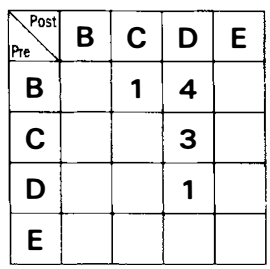

Disc herniation $(n=9)$

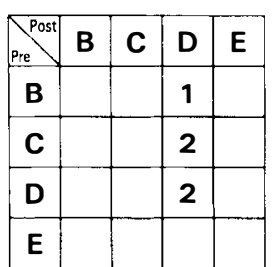

OPLL $(n=5)$

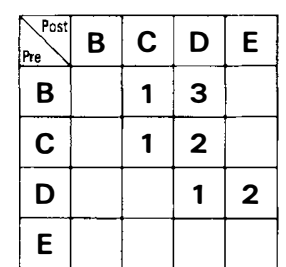

Canal stenosis $(n=10)$
Figure 3 Relationship between neurological recovery and canal narrowing factors
The effects of surgical treatment were compared between surgery done in the early stage and in the late stage after injury. The preoperative neurological state was assessed using Frankel grade just prior to surgery. In early stage surgery group, all of the patients improved neurologically, whereas $10 \quad(55.6 \%)$ remained unchanged in the late stage surgery group $(P<0.01)$.

\section{Case reports}

Case 1

Whilst working, a 48-year-old man fell from a height of 2 meters after receiving an electrical shock. On admission, his Frankel assessment was grade C. A myelogram showed a complete block at the $\mathrm{C} 4 / \mathrm{C} 5$ level, and a CT myelogram also showed anterior spinal cord compression at the C4/C5 level (Figure 4a). Both a discogram and a CT discogram showed leakage of the contrast medium into the epidural space at $\mathrm{C} 4 / 5$ and C5/6 (Figure 4b). Disc herniation at the $\mathrm{C} 4 / \mathrm{C} 5$ level was seen in the sagittal MRI T1-weighted image (Figure 4c). Because the patient had not improved satisfactorily in spite of undergoing skull tong traction, 6 weeks after injury, anterior decompression and spinal fusion were performed at $\mathrm{C} 4 / \mathrm{C} 5$ and $\mathrm{C} 5 / \mathrm{C} 6$ (Figure 4d). Three months later, he was able to walk without aid and to eat with a spoon. His Frankel assessment was grade $\mathrm{D}$ at the follow-up.

Case 2

Subsequent to falling and striking her forehead, a 47year-old woman had immediate difficulty in moving her upper and lower extremities and had urinary retention. She was admitted to a hospital and treated conservatively. Because she did not show satisfactory improvement, 5 weeks after injury she was transferred to our hospital. Her Frankel assessment was grade D on admission. Plain radiographs and a conventional tomogram showed ossification of the posterior longitudinal ligament (OPLL) from the C2 to C6 level (Figure 5a). This is the mixed type in the OPLL classification. The space-occupying ratio of the OPLL was $53 \%$ at the $\mathrm{C} 4$ level. A myelogram and CT myelogram disclosed a ventral extradural defect from C3 to C6 and anterior spinal cord compression (Figure 5b). Expansive open door laminoplasty was performed from $\mathrm{C} 3$ to $\mathrm{C} 78$ weeks after injury. Her postoperative myelogram and CT myelogram showed an enlarged spinal canal and expansion of the spinal cord (Figure 5c). Sensory disturbance and hand clumsiness had lessened, but the Frankel assessment was still D when she was last seen 2 years after surgery.

\section{Discussion}

Very few studies to our knowledge ${ }^{1,4}$ have dealt with the prognosis for neurological improvement, or the 

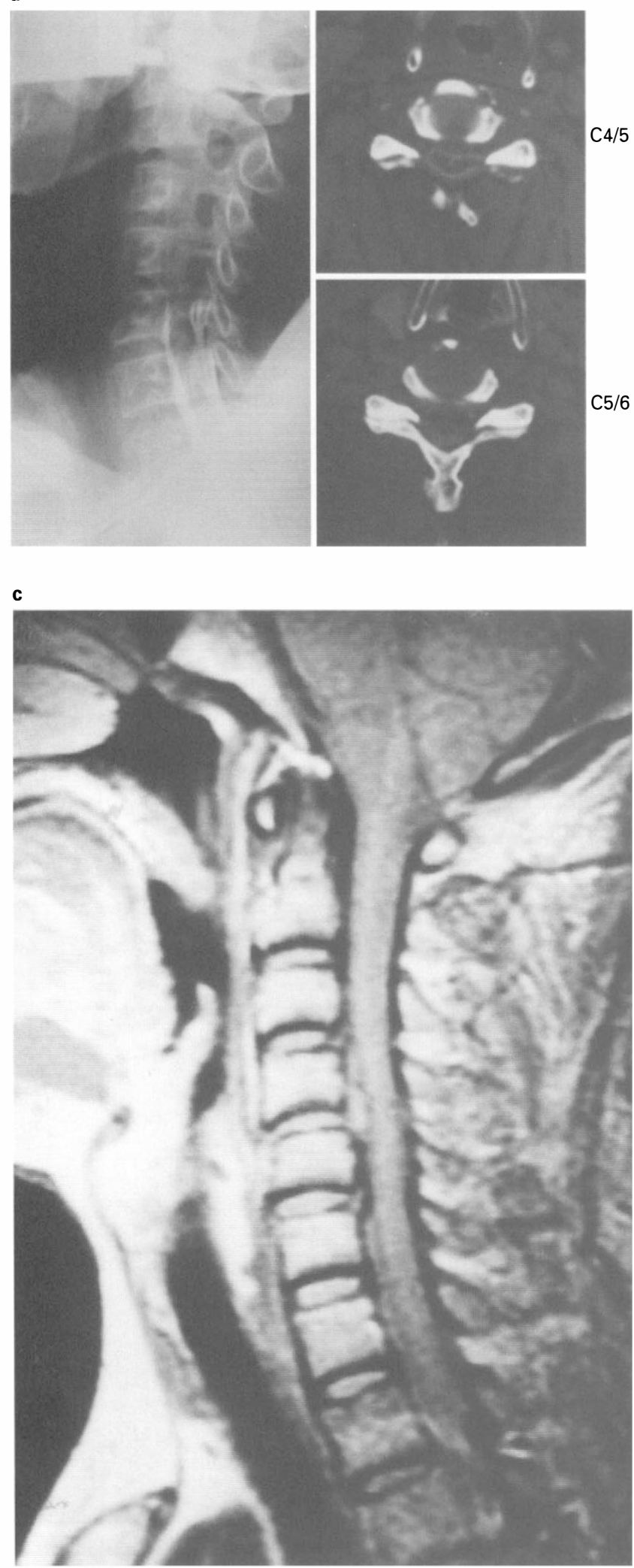

b

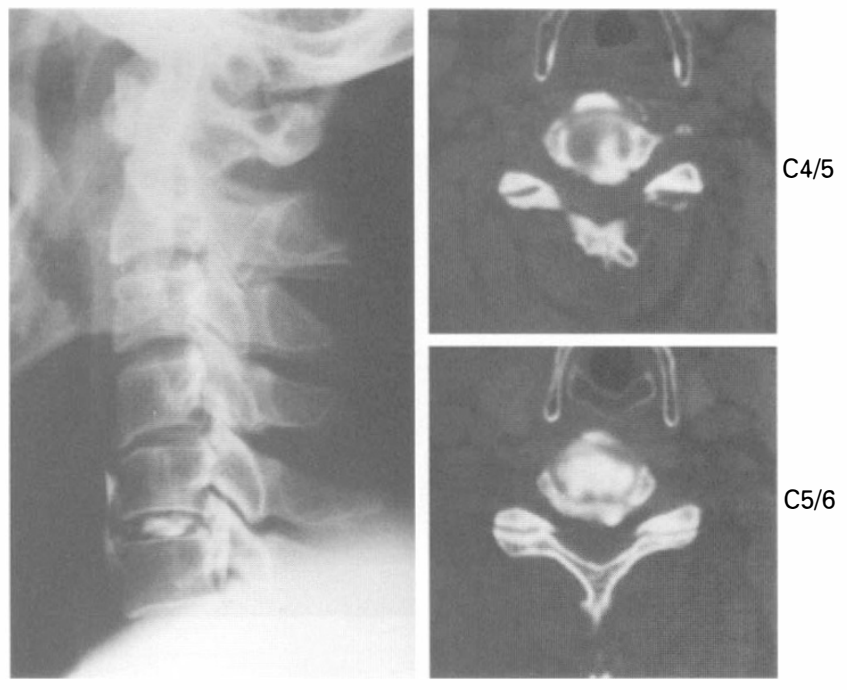

d

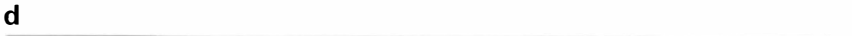

Figure 4 (a) A 48-year-old man with traumatic cervical disc herniation. The myelogram shows complete block at C4/5 level. CT myelogram shows anterior spinal cord compression. (b) Discogram and CT discogram showing leakage of the contrast medium into the epidural space at $\mathrm{C} 4 / 5$ and $\mathrm{C} 5 / 6$. (c) Disc herniation at $\mathrm{C} 4 / 5$ level is seen in the sagittal MRI Tl-weighted image. (d) Post operative X-ray (after 6 years). Complete bony union was obtained C4-6 


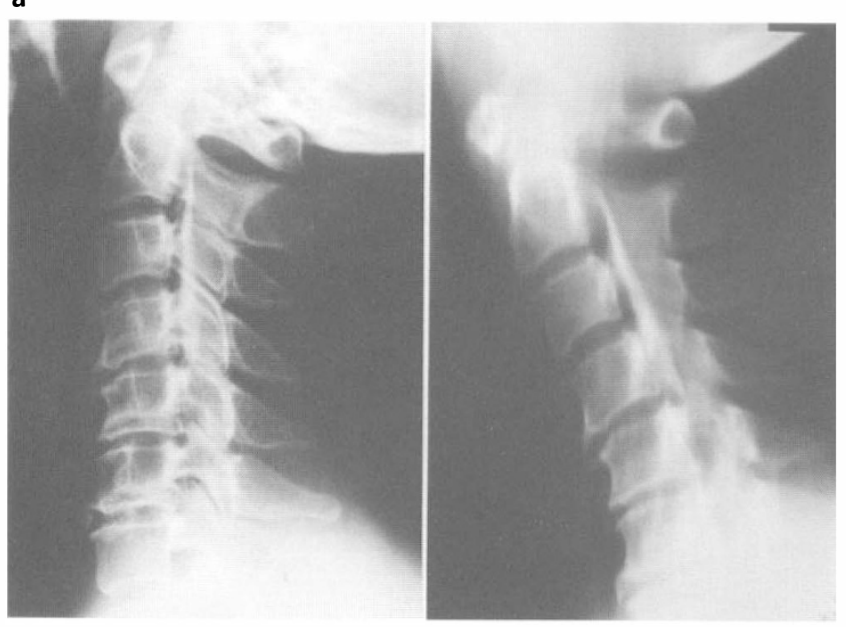

b

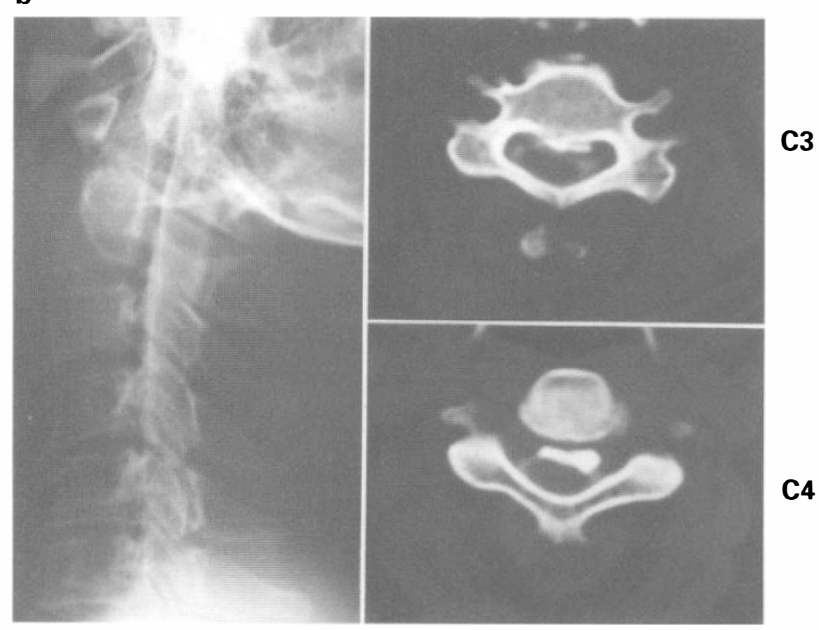

c

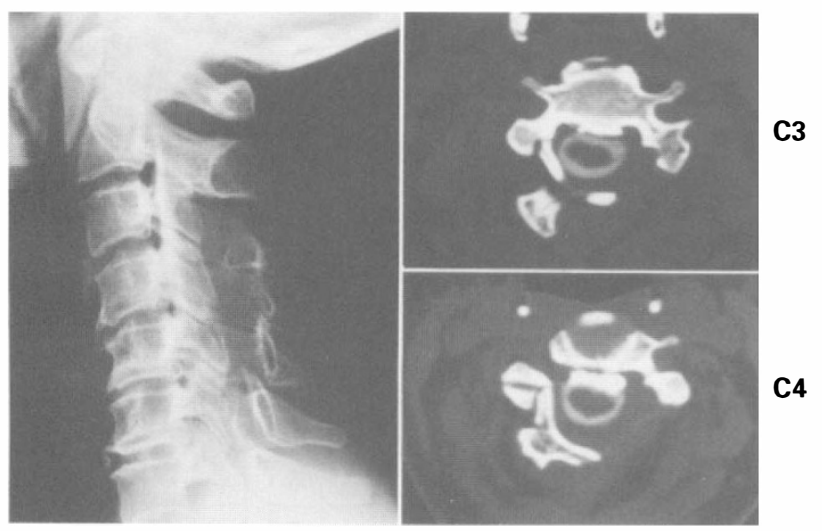

Figure 5 (a) A 47-year-old woman, with OPLL from C2 to C6. A plain radiograph and tomogram. (b) The myelogram and $\mathrm{CT}$ myelogram revealed ventral extradural defect from C3 to C6 and anterior spinal cord compression. (c) Postoperative CT myelogram showing the enlarged spinal canal and expansion of the spinal cord correlations between the degree of restoration, the mechanism of injury, neurological deficit in the intitial stage, and differences in treatment. Many factors may affect the prognosis for the neurological recovery of patients with an incomplete cervical SCI. Age, the magnitude of the trauma, pre-existing canal narrowing factor and decompressive surgery have been considered. Bohman et $a l^{4}$ reported that surgical results were not as good for patients who had an extension injury compared to those who had another mechanism of injury, although our study showed no difference between those with flexion and extension injuries. According to their study, there were no correlations between age, the magnitude of the injury and neurological improvement. In our study, neurological recovery was not good for older aged patients although the magnitude of the injury did not influence the clinical results.

Several canal narrowing factors which may affect neurological recovery in incomplete cervical spinal cord injuries were recognized: bursting fracture, facet dislocation, posterior osteophytes, disc herniation, OPLL and developmental canal stenosis. Murone et $a l^{5}$ suggested that the risk of myelopathy in spondylosis increases when the sagittal diameter in less than $12 \mathrm{~mm}$. We also defined cervical canal stenosis as below $12 \mathrm{~mm}$ in A-P diameter of the spinal canal. Torg et $a l^{6}$ detected 17 patients with developmental canal stenosis out of 32 patients of the neurapraxia of the cervical spinal cord with transient tetraplegia. Wagner et $a l^{7}$ found that there was radiological evidence of preexisting spondylosis in two patients out of three patients who did not recover to the extent that would have been expected. As the

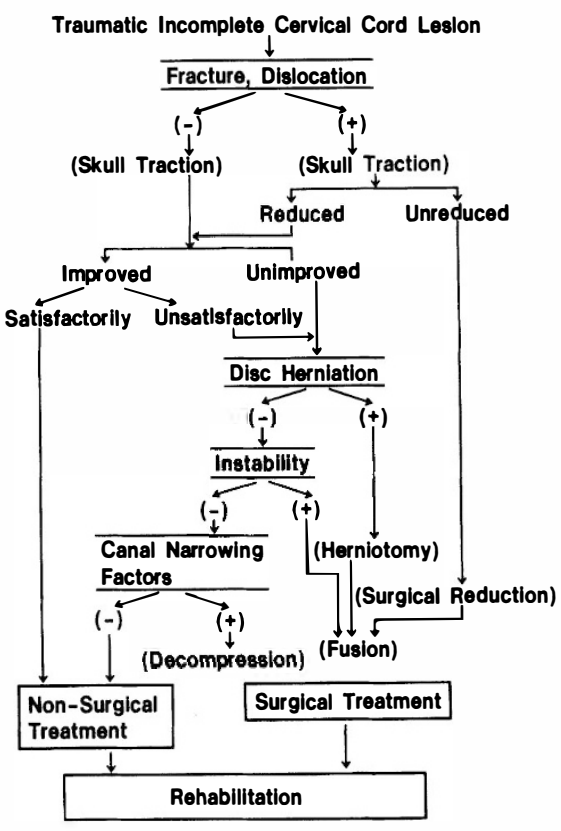

Figure 6 Flowchart of treatments for traumatic incomplete cervical spinal cord injury 
results of this study showed, paralysis is expected to lessen when the spinal cord is compressed by soft material such as a disc herniation. In fact, Raynor et $a l^{8}$ reported that surgical excision of the disc produced rapid neurological improvement in half of the patients with cervical cord compression secondary to acute disc protrusion from trauma.

Controversy exists about operative versus nonoperative treatment in patients with an incomplete cervical SCI. Some studies recommend early surgical decompression, 9,10 others advocated conservative management. ${ }^{11-14}$ There has not been a clear consensus in previous publications on the management of this lesion. Schneider et $a l^{11}$ stated that neurological recovery was a function of the pathophysiological process, whether or not intramedullary edema or hematomyelia existed, and because the overall prognosis generally was favorable early surgery was not recommended. According to Hamel et al ${ }^{15}$ there was no significant difference between those who were treated conservatively and those treated surgically in the series of patients with an incomplete transection syndrome. Harris et $a l^{16}$ concluded that early operation did not influence the neurological outcome, and that reduction of a fracture-dislocation was not essential for neurological recovery. Maynard et al $^{17}$ also indicated that surgery undertaken within 4 weeks of injury did not enhance neurological recovery. Surgery is not undertaken in patients with incomplete lesions who are improving. ${ }^{18}$ In our study, all of the patients who underwent early stage surgery improved at least one grade; whereas, the condition of 10 of the patients $(55.6 \%)$ who underwent late stage surgery remained unchanged. Thus we recommend early stage surgery when neurological recovery cannot be observed within a few weeks after the injury because it is not possible to conclude that decompression is without effect on the neurological outcome in the absence of a control group of patients whose spinal canals remained narrowed at follow-up review. ${ }^{7}$

A flowchart of the treatments used for incomplete cervical spinal cord injury is given in Figure 6. When the fracture or dislocation cannot be reduced by skull traction, surgical reduction and spinal fusion are recommended. Surgical treatment is indicated for patients without fracture or dislocation when there is no neurological improvement or when it is unsatisfactory. In such cases, anterior decompression and spinal fusion is recommended if disc herniation is detected, and anterior spinal fusion if cervical instability remains. Posterior decompression (ELAP) is indicated if canal narrowing factors such as OPLL exist. Wagner et $a l^{7}$ described that the findings of the present study of the early decompression and neurological outcome in acute cervical SCI indicate that the initial injury to the cervical spinal cord and spine remains the primary determinant of the neurological outcome. Therefore, a meticulous neurological examination such as Heiden et $a l^{19}$ described is required to determine the type of incomplete cervical SCI that is to be treated.

\section{Conclusions}

(1) Thirty-seven $(82.2 \%)$ of 45 patients showed neurological improvement of at least one grade.

(2) Patients with disc herniation improved better than those with OPLL.

(3) There were no correlations between the mechanism of the injury, the magnitude of the injury and neurological improvement.

(4) Patients who underwent the early stage surgery improved better than those who had the late stage surgery.

\section{References}

1 Usui H, Hirabayashi K. Acute central cervical cord injury. Seikeigeka 1981; 32: 1803-1812 (in Japanese).

2 Hirabayashi K, Satomi K. Operative procedure and results of expansive open-door laminoplasty. Spine 1988; 13: $870-879$.

3 Frankel HL, Hancock DO, Melzak J. The value of postural reduction in the intitial management of closed injuries of the spine with paraplegia and tetraplegia. Paraplegia 1969; 7: 179192.

4 Bohman $\mathrm{HH}$, Andreson PA. Anterior decompression and artrodesis of the cervical spine: Long-term motor improvement. JBJS 1992; 74A: $671-682$.

5 Muroe I. The importance of the sagittal diameters of the cervical spinal canal in relation to spondylosis and myelopathy. JBJS 1974: 56B: $30-36$.

6 Torg JS et al. Neurapraxia of the cervical spinal cord with transient quadriplegia. JBJS 1986; 68A: $1354-1370$.

7 Wagner FC Jr, Chehrazi B. Early decompression and neurological outcome in acute cervical spinal cord injuries. $J$ Neurosurg 1982; 56: $699-705$.

8 Raynor RB. Cervical cord compression secondary to acute disc protrusion in trauma. Incidence and response to decompression. Spine 1977; 2: $39-43$.

9 Evans JP, Posenaur A. Spinal cord injuries. A 15-year survey. Arch Surg 1956; 72: 812-816.

10 Brodkey JS. The syndrome of acute central cervical spinal cord injury. Surg Neurol 1980; 14: $251-257$.

11 Schneider RC. The syndrome of acute anterior cervical spinal cord injury. J Neurosurg 1955; 12: $95 \cdots 122$.

12 Bedbrook G. Pathological principles in the management of spinal trauma. Paraplegia 1966; 4: 43 - 56.

13 Benassy $\mathbf{J}$, Blanchard J, Lecoq P. Neurological recovery in paraand tetraplegia. Paraplegia 1967; 4: 259- 261.

14 Meinecke FW. Early treatment of traumatic paraplegia. Paraplegia 1964; 1: $262-270$.

15 Hamel E et al. Results of conservative and surgical early treatment of cervical spine injuries. In: Wullenweber R, Brock M, Hamer J (eds). Lumbar Disc Adult Hydrocephalus. vol 4. Springer-Verlag: Berlin/Heidelberg/New York, 1977, pp. $185-$ 190.

16 Harris P et al. The prognosis of patients sustaining severe cervical spine injury (C2-C7 inclusive). Paraplegia 1980; 18: $324-330$.

17 Maynard FM et al. Neurological prognosis after traumatic quadriplegia. Three-year experience of California regional spinal cord injury care system. J Neurosurg 1979; 50: 611-616.

18 Wagner FC Jr, Chehrazi B. Spinal cord injury. Surg Clin North Am 1980; 60: 1049 1054

19 Heiden JS, Weiss MH, Rosenberg AW. Management of cervical spinal cord trauma in Southern California. J Neurosurg 1975; 43: $723-736$. 